CONFORMAL GEOMETRY AND DYNAMICS

An Electronic Journal of the American Mathematical Society

Volume 1, Pages 104-111 (December 16, 1997)

S 1088-4173(97)00013-1

\title{
UNIFORMLY QUASIREGULAR MAPPINGS OF LATTÈS TYPE
}

\author{
VOLKER MAYER
}

\begin{abstract}
Using an analogy of the Lattès' construction of chaotic rational functions, we show that there are uniformly quasiregular mappings of the $n$ sphere $\overline{\mathbb{R}}^{n}$ whose Julia set is the whole sphere. Moreover there are analogues of power mappings, uniformly quasiregular mappings whose Julia set is $\mathbb{S}^{n-1}$ and its complement in $\mathbb{S}^{n}$ consists of two superattracting basins. In the chaotic case we study the invariant conformal structures and show that Lattès type rational mappings are either rigid or form a 1-parameter family of quasiconformal deformations.
\end{abstract}

\section{INTRODUCTION}

There are a number of important tools, such as Rickman's quasiregular version of Montel's theorem, that allow the development of a quasiregular analogue of the theory of complex dynamical systems. However it is not clear how rich such a theory is. What kind of uniformly quasiregular mappings with non-empty branch set and interesting dynamical behaviour exist? Recall that in dimension $n \geq 3$ by Liouville's theorem (see e.g. [Ri]) every 1-quasiregular map is a Möbius transformation and that, furthermore, there is a rigidity phenomenon: there is a constant $K_{0}>1$ so that every $K$-quasiregular map with $K \leq K_{0}$ is a homeomorphism.

Examples of uniformly quasiregular mappings are elements of quasiconformal groups. Such groups have been studied and there are now many interesting examples in the literature. But these mappings are homeomorphic, their dynamical behaviour is elementary, and their Julia set $J(f)$ consists of at most two points.

Iwaniec and Martin [IM] gave the first examples of uniformly quasiregular maps with interesting dynamical behaviour and with non-empty branch set. The Julia sets of their examples are Cantor sets; the Fatou sets are attracting basins.

In $1918 \mathrm{~S}$. Lattès gave the first example of a rational map $f$ of $\hat{\mathbb{C}}$ which is chaotic, i.e. the Julia set is the whole sphere $J(f)=\hat{\mathbb{C}}$. The geometric nature of Lattès' construction adapts to our higher-dimensional setting and leads to chaotic uniformly quasiregular mappings of $\overline{\mathbb{R}}^{n}$.

Theorem 1. For every $d=2,3, \ldots$ there is a uniformly quasiregular map $f$ of $\overline{\mathbb{R}}^{n}$ with degree $d^{n}$ whose Julia set is the whole sphere $J(f)=\overline{\mathbb{R}}^{n}$.

We study the associated invariant conformal structures of these mappings (the existence of such structures is proved in [IM]). Theorem 3 is an $n$-dimensional version of the fact that Lattès rational mappings are either rigid or form a 1parameter family of quasiconformal deformations.

Received by the editors March 7, 1997, and, in revised form, September 22, 1997.

1991 Mathematics Subject Classification. Primary 30C65; Secondary 58Fxx.

(c)1997 American Mathematical Society 
Lattès' construction also leads to uniformly quasiregular maps of $\overline{\mathbb{R}}^{n}$ which behave like the polynomial $z \mapsto z^{d}$. We only present the three-dimensional case here. There are quasiregular mappings that behave like power mappings [MRV, Ri]. Our mappings are uniformly quasiregular and the dynamical behaviour is also like that of $z^{d}$. Moreover, the construction that leads to these mappings gives, in dimension $n=2$, precisely the power mappings. So we propose the following mappings as the natural counterparts of power mappings.

Theorem 2. For every $d=2,3, \ldots$ there is a uniformly quasiregular map $f$ of $\overline{\mathbb{R}}^{3}$ with degree $d^{2}$, with Julia set $J(f)=\mathbb{S}^{2}$ and whose Fatou set $F(f)=\overline{\mathbb{R}}^{3} \backslash \mathbb{S}^{2}$ consists of two superattracting basins. Moreover, the map $f$ can be chosen to be an extension of a Lattès rational map.

The construction of this example goes as follows. Denote by $\left.W^{n}=\right] 0,1\left[{ }^{n}\right.$ the unit cube of $\mathbb{R}^{n}$ and by $Z=W^{2} \times \mathbb{R}$ the standard cylinder of $\mathbb{R}^{3}$. Choose a quasiconformal homeomorphism $h$ from $W^{n}$ onto the upper half-space of $\mathbb{R}^{n}$ in the case of the chaotic example, and from the cylinder onto the upper half-space of $\mathbb{R}^{3}$ for the maps that behave like $z^{d}$. Now extend $h$ to a quasimeromorphic map defined on $\mathbb{R}^{n}$ by using reflections. So $h$ alternately maps cubes or cylinders to the upper and lower half-spaces and it is automorphic with respect to the affine group whose elements correspond to "taking an even number of reflections in faces". In semi-conjugating the multiplication $x \mapsto d x$ we get our maps $f$ :

$$
f(h(x))=h(d x) \quad \text { for } \quad x \in \mathbb{R}^{n} .
$$

\section{Definitions, AUTOMORPhic MAPPings AND SUPERATTRACTING FIXED POINTS}

In this section we recall the definition of quasiregular mappings, some basic facts of iteration and the automorphic quasimeromorphic mappings that we need.

2.1. Quasiregularity. Let $D \subset \mathbb{R}^{n}$ be a domain and $f: D \rightarrow \mathbb{R}^{n}$ a mapping of Sobolev class $W_{l o c}^{1, n}(D)$. We consider only orientation preserving mappings, that is, the Jacobian determinant $J_{f}(x) \geq 0$ for a.e. $x \in D$. Such a mapping is said to be $K$-quasiregular, where $1 \leq K<\infty$, if

$$
\max _{|h|=1}\left|f^{\prime}(x) h\right| \leq K \min _{|h|=1}\left|f^{\prime}(x) h\right| \quad \text { for } \quad \text { a.e. } \quad x \in D .
$$

The smallest number $K$ for which the above inequality holds is called the linear dilatation of $f$. We will also make use of the inner and outer dilatations $K_{I}, K_{O}$ as defined in [Ri]. A non-constant quasiregular mapping can be redefined on a set of measure zero so as to make it continuous, open and discrete, and we shall always assume that this has been done. If $D$ is a domain in the compactification $\overline{\mathbb{R}}^{n}$ equipped with the spherical metric, then we use the chart at infinity $x \mapsto x /|x|^{2}$ to extend in the obvious manner the notion of quasiregularity to mappings $f$ : $D \rightarrow \overline{\mathbb{R}}^{n}$. Such mappings are also said to be quasimeromorphic. A mapping $f$ of a domain $D$ into itself is called uniformly quasiregular if there is some $1 \leq K<\infty$ such that all the iterates $f^{k}$ are $K$-quasiregular. The branch set $B_{f}$ is the set of points $x \in D$ for which $f$ is not locally homeomorphic at $x$. 
2.2. Basic facts of iteration. We consider uniformly quasiregular mappings of $\overline{\mathbb{R}}^{n}$. The dynamical behaviour of such a mapping splits the sphere into two parts: the Fatou set $F(f)$, which is the set of points $x$ for which $\left\{f^{k}\right\}$ is a normal family in a neighborhood of $x$, and the Julia set $J(f)=\overline{\mathbb{R}}^{n} \backslash F(f)$. The Fatou set is open, the Julia set is closed and they are both completely invariant. Moreover, if the mapping $f$ has a non-empty branch set, then the Julia set cannot be empty. See [IM] for this and for a more detailed presentation.

Fixed points which are also branch points will be called superattracting fixed points. From the local behaviour of quasiregular mappings, it follows that superattracting fixed points are attracting. This is the content of the next Lemma. In particular, it follows that in a neighborhood of a superattracting fixed point $x$ the iterates $f^{k}$ converge uniformly to $x$. Let $\Omega_{x}=\left\{y \in \overline{\mathbb{R}}^{n} ; f^{k}(y) \rightarrow x\right\}$ be the basin of attraction of $x$ and $\Omega_{x}^{*}$ the component of $\Omega_{x}$ containing $x$. When $x$ is completely invariant, that is, if $f(x)=f^{-1}(x)=\{x\}$, then the immediate basin of attraction $\Omega_{x}^{*}$ coincides with $\Omega_{x}$.

Lemma 2.1. Let $x$ be a superattracting fixed point of the uniformly quasiregular mapping $f$ of $\overline{\mathbb{R}}^{n}$. Then there exists $k \in \mathbb{N}$ and $R>0$ such that

$$
f^{k}(\bar{B}(x, r)) \subset B\left(x, \frac{r}{2}\right) \quad \forall 0<r<R .
$$

Proof. We may assume that the fixed point is the origin. We use the notation of [Ri]. Assume for a moment that we have the following relation concerning the local index:

$$
i\left(0, f^{k}\right)=i(0, f)^{k}, \quad k \in \mathbb{N} .
$$

By [Ri, Thm. III.4.7] there exists for every $k \in \mathbb{N}$ positive constants $\rho$ and $C$ such that

$$
\left|f^{k}(y)\right| \leq C|y|^{\alpha} \quad \forall y \in B_{\rho}=B(0, \rho)
$$

with $\alpha=\left(i(0, f)^{k} / K_{I}\right)^{\frac{1}{n-1}}$ and where $K_{I}$ is the inner dilatation of $f^{k}$. The fixed point 0 is supposed to be also a branch point and so $i(0, f)>1$. It suffices now to choose $k$ such that $\alpha>1$. The Lemma holds then for $R \in] 0, \rho\left[\right.$ with $C R^{\alpha-1}<1 / 2$.

It remains to prove (2). Let $s=s(k)>0$ so that for any $j=1, \ldots, k$ the component $U_{j}$ of $\left(f^{j}\right)^{-1}\left(B_{s}\right)$ containing 0 is contained in $B_{1}$ and that $\overline{U_{j}} \cap\left(f^{j}\right)^{-1}(0)=\{0\}$. These components are normal domains, i.e. $f\left(\delta U_{j}\right)=\delta f\left(U_{j}\right)$ and $f\left(U_{j+1}\right)=U_{j}$ [Ri, Lemma I.4.7]. When $F$ is a $K$-quasiregular mapping the Hausdorff dimension of the image of the branch set is strictly less then $n$ [Sa]. Hence, we can choose $y \in B_{s} \backslash f^{k}\left(U_{k} \cap B_{f^{k}}\right)$. Notice that any preimage $x \in\left(f^{j}\right)^{-1}(y) \cap U_{j}$ is in the set $x \in U_{j} \backslash f\left(U_{j+1} \cap B_{f}\right)$. This enables us to apply [Ri, Prop. I.4.10] to get $i\left(0, f^{k}\right)=\operatorname{card}\left(f^{k}\right)^{-1}(y) \cap U_{k}$ and $i(0, f)=\operatorname{card}(f)^{-1}(x) \cap U_{j+1}$ for any $x \in\left(f^{j}\right)^{-1}(y) \cap U_{j}$ and $j=0, \ldots, k-1$. From this the relation (2) immediately follows.

2.3. Automorphic quasimeromorphic mappings. Recall that a map $h$ is automorphic with respect to a group $\Gamma$ of Möbius transformations if $h \circ \gamma=h$ for every $\gamma \in \Gamma$. The following facts about automorphic quasimeromorphic mappings that we need are found in [Ri]. 
2.3.1. Zorich's mapping. There is a quasiregular mapping $h: \mathbb{R}^{3} \rightarrow \mathbb{R}^{3} \backslash\{0\}$ automorphic with respect to

$$
\Gamma_{1}=\left\langle x \mapsto\left(-x_{1},-x_{2}, x_{3}\right) ; x \mapsto x+2 e_{1} ; x \mapsto x+2 e_{2}\right\rangle .
$$

The construction of $h$ goes as follows. First map $Z=W^{2} \times \mathbb{R}$ by radial stretching in the planes $\mathbb{R}^{2} \times\left\{x_{3}\right\}$ onto the round cylinder $\mathbb{D} \times \mathbb{R}$. The round cylinder $\mathbb{D} \times \mathbb{R}$ can be mapped quasiconformally onto the upper 3-dimensional half-space $\mathbb{H}$ by the mapping $\left(r, \varphi, x_{3}\right) \mapsto(t, \varphi, \theta)$ with $t=e^{x_{3}}, \theta=\pi \frac{r}{2}$, where cylindrical and spherical coordinates, respectively, are used. Now $h$ is obtained by extending this map to a quasiregular map of $\mathbb{R}^{3}$ onto $\mathbb{R}^{3} \backslash\{0\}$ using reflections on faces of $Z$ and in $\partial \mathbb{H}$ in the range. So $h$ alternately maps cylinders onto the upper and lower half-space. Note that $\Gamma_{1}$ is precisely the group whose elements are formed from an even number of reflections in faces of $Z$. The branch set of $h$ is $B_{h}=\mathbb{Z}^{2} \times \mathbb{R}$, the edges of $Z$ and all the "reflected" cylinders.

The $n$-dimensional analogue of Zorich's mapping [MS, Ri] can be used to get an $n$-dimensional version of our Theorem 1.

2.3.2. Quasimeromorphic analogue of the Weierstrass P-function. The following example of a quasimeromorphic mapping that behaves like the Weierstrass $P$ function is due to Martio and Srebro [MS].

We start here with the unit cube $W^{n}$. Using again radial stretching and a translation we get a bilipschitz mapping from $W^{n}$ onto the unit ball $\mathbb{B}^{n}$. So there is a quasiconformal map from $W^{n}$ onto the upper half-space $\mathbb{H}^{n}$. Now one extends this map as in Zorich's example by using reflections on the faces of $W^{n}$ and on $\partial \mathbb{H}^{n}$. In this way we get a quasimeromorphic map $h: \mathbb{R}^{n} \rightarrow \overline{\mathbb{R}}^{n}$ which maps cubes alternately onto the upper and lower half-spaces. This map is automorphic with respect to the affine group, say $\Gamma_{2}$, corresponding to the even number of reflections on the faces of $W^{n}$. The group $\Gamma_{2}$ is generated by the translations $x \mapsto x+2 \nu$, $\nu \in \mathbb{Z}^{n}$, and by the elements of the group

$$
\Sigma=\left\langle x \mapsto\left(x_{1}, \ldots, x_{i-1},-x_{i}, x_{i+1}, \ldots, x_{j-1},-x_{j}, x_{j+1}, \ldots, x_{n}\right) ; 1 \leq i<j \leq n\right\rangle .
$$

The branch set of $h$ consists in

$$
B_{h}=\bigcup_{i \neq j} \bigcup_{k, l \in \mathbb{Z}}\left\{x_{i}=k ; x_{j}=l\right\} .
$$

\section{The ChaOtic Uniformly QUASIREgUlar LATtès TYPe MAPPINGS}

The chaotic uniformly quasiregular mappings that we look for are the solutions of Schröders equation (1) where $h$ is the quasimeromorphic analogue of Weierstrass' $P$-function. For this we have to check that the Schröder equation (1) gives a well defined map $f$. This is based on the fact that the multiplication $x \mapsto d x$ is compatible with the reflections.

Let $A(x)=\lambda U x$, with $\lambda>0$ and $U \in S O(n)$, be a conformal affine map. We suppose that $A$ is compatible with the reflections: if $x$ is in some cube $W^{\prime}$ and if we reflect it an even number of times in its faces, we get another cube $W^{\prime \prime}$ containing $x^{\prime}$, the image of $x$ under these reflections. This is an equivalence relation $\simeq$ and we suppose that $A(x) \simeq A\left(x^{\prime}\right)$ whenever $x \simeq x^{\prime}$. In terms of the group $\Gamma_{2}$ this means that if $x^{\prime}=\gamma(x), \gamma \in \Gamma_{2}$, then $A\left(x^{\prime}\right)=\gamma^{\prime} A(x)$ for some $\gamma^{\prime} \in \Gamma_{2}$. So we demand that $A \circ \Gamma_{2} \circ A^{-1} \subset \Gamma_{2}$. Note that in this case $A$ automatically preserves the group 
of translations $\left\{x \mapsto x+2 \nu ; \nu \in \mathbb{Z}^{n}\right\}$, which we note simply $2 \mathbb{Z}^{n}$, and also the group $\Sigma$ :

$$
A \circ 2 \mathbb{Z}^{n} \circ A^{-1} \subset 2 \mathbb{Z}^{n} \text { and } A \circ \Sigma \circ A^{-1}=\Sigma .
$$

Therefore, we can first project $A$ to a map $m_{A}$ of the torus $\mathbb{T}=\mathbb{R}^{n} / 2 \mathbb{Z}^{n}$ and, in a second step, to a map $M_{A}$ of $\mathbb{R}^{n} / \Gamma_{2}=\mathbb{T} / \tilde{\Sigma}$, where $\tilde{\Sigma}$ is the projection of $\Sigma$ onto $\mathbb{T}$.

On the other hand, our quasimeromorphic Weierstrass' $P$-function $h$ is $\Gamma_{2}$ automorphic. So $h$ induces a homeomorphic map $\tilde{h}: \mathbb{R}^{n} / \Gamma_{2} \rightarrow \overline{\mathbb{R}}^{n}$. Hence, when $A$ is a given map compatible with the reflections, then $f_{A}=\tilde{h} \circ M_{A} \circ \tilde{h}^{-1}$ is a solution of Schröders' equation

$$
f_{A} \circ h(x)=h \circ A(x) \quad \text { for } \quad x \in \mathbb{R}^{n} .
$$

In particular, every multiplication $x \mapsto d x(d=2,3, \ldots)$ gives rise to a solution $f_{d}$.

Lemma 3.1. The solution of Schröders' equation $f_{A}$ is uniformly quasiregular.

Proof. Recall that $h$ is obtained by extending a quasiconformal map defined on $W^{n}$ with range the upper half-space $\mathbb{H}^{n}$. This quasiconformal map is in fact the composition of a Möbius transformation with the radial stretching map that maps $W^{n}$ onto the unit ball $\mathbb{B}^{n}$. The radial stretching map is bilipschitz up to the boundary which allows us to check that $f_{A} \in W^{1, n}\left(\overline{\mathbb{R}}^{n}\right)$ and that $f_{A}$ is continuous.

The set $h\left(B_{h}\right)$ of critical values of $h$ consists in a finite number of smooth hypersurfaces of $\mathbb{R}^{n-1} \cup\{\infty\}$. For every $x \in \overline{\mathbb{R}}^{n} \backslash h\left(B_{h}\right)$ there exists an open neighborhood $U_{x}, x \in U_{x}$, so that the restriction of $h$ to any component of $h^{-1}\left(U_{x}\right)$ is a homeomorphism. Hence, we can choose an inverse branch of $h$, say $H$, defined on $U_{x}$, with

$$
f^{k}\left(x^{\prime}\right)=h\left(A^{k} H\left(x^{\prime}\right)\right) \quad \text { for every } \quad x^{\prime} \in U_{x} .
$$

This shows that $f$, and all its iterates $f^{k}$, are $K^{2}$-quasiregular where $K$ is the constant of quasiregularity of $h$.

Lemma 3.2. The Julia set of the map $f_{A}$ is $J\left(f_{A}\right)=\overline{\mathbb{R}}^{n}$.

Proof. The proof is the same as for rational maps. The origin is a repelling fixed point for $A$. Consider the $\Gamma_{2}$-orbit of it: $\Gamma_{2}(0)=\left\{\gamma(0) ; \gamma \in \Gamma_{2}\right\}=2 \mathbb{Z}^{n}$. The set $E=\bigcup_{k>0} A^{-k}\left(\Gamma_{2}(0)\right)$ is a dense subset of $\mathbb{R}^{n}$. Hence, $h(E)$ is a dense subset of $\overline{\mathbb{R}}^{n}$ and it is the backward orbit under $f_{A}$ of the repelling fixed point $h(0)$ of $f_{A}$. We conclude that $\left(f_{A}^{k}\right)$ cannot be equicontinuous in a neighborhood of any point of $\overline{\mathbb{R}}^{n}$. This means that the Julia set is the whole sphere.

Lemma 3.3. The branch set $B_{f}$ of the mapping $f=f_{A}$ is contained in $h \circ$ $A^{-1}\left(B_{h}\right)$. Moreover, every non-critical value $y \in \overline{\mathbb{R}}^{n} \backslash f\left(B_{f}\right)$ has precisely $\lambda^{n}$ preimages.

Proof. We first prove the affirmation concerning the branch set $B_{f}$. If $x \notin h \circ$ $A^{-1}\left(B_{h}\right)$, so if $x=h(a)$ with $a \notin A^{-1}\left(B_{h}\right)$, then $y=f(x)=h \circ A(a) \notin h\left(B_{h}\right)$. In a neighborhood $U_{y}$ of $y$ we can choose $H$, an inverse branch of $h$, with $A(a)=H(y)$. Therefore, $h \circ A^{-1} \circ H$ is an inverse branch of $f$ defined in $U_{y}$. This means that $x \notin B_{f}$.

In order to determine the degree of $f$ it is convenient to work in the quotient space $\mathbb{R}^{n} / \Gamma_{2}$. We proceed in two steps. First let us see what happens on the torus $\mathbb{T}$. It is clear that the preimage of any $q \in \mathbb{T}$ under $m_{A}$ consists in precisely $\lambda^{n}$ points. Now, 
when we do the second projection $\pi: \mathbb{T} \rightarrow \mathbb{T} / \tilde{\Sigma}=\mathbb{R}^{n} / \Gamma_{2}$, then $M_{A}^{-1}(\pi(q))$ consists also in $\lambda^{n}$ points, provided $m_{A}^{-1}(q)$ has no $\tilde{\Sigma}$-equivalent points. This is true for points $q \in \mathbb{T}$ whose liftings to $\mathbb{R}^{n}$ are not in $A\left(B_{h}\right)$. Therefore, each $y \notin h \circ A\left(B_{h}\right)$ has precisely $\lambda^{n}$ preimages. If $y \in h \circ A\left(B_{h}\right) \backslash f\left(B_{f}\right)$, then $f$ is homeomorphic in a neighborhood $U_{x}$ of each point $x \in f^{-1}(y)$. Let $V=\bigcap_{x \in f^{-1}(y)} f\left(U_{x}\right)$ and choose $y^{\prime} \in V \backslash h \circ A\left(B_{h}\right)$. Since this point $y^{\prime}$ has $\lambda^{n}$ preimages we conclude that the same is true for $y$.

\section{INVARIANT CONFORMAL STRUCTURES}

A conformal structure is a measurable map $\mu$ from $\overline{\mathbb{R}}^{n}$ into the space $S=$ $S L(n, \mathbb{R}) / S O(n)$ of $n \times n$ real positive definite matrices with determinant 1 . The general linear group $G L(n, \mathbb{R})$ acts transitively on the right of $S$ by

$$
X[M]=|\operatorname{det} X|^{-\frac{2}{n}} X^{T} M X, \quad X \in G L(n, \mathbb{R}), \quad M \in S .
$$

The Riemannian metric $d s^{2}=\operatorname{tr}\left(M^{-1} d M\right)^{2}$ on $S$ gives rise to a metric which is invariant under the right action of $G L(n, \mathbb{R})$. We denote this distance by $\operatorname{dist}(A, B)$, $A, B \in S$. More information about this can be found for example in [Tu, IM].

A conformal structure $\mu$ is $f$-invariant if for a.e. $x \in \overline{\mathbb{R}}^{n}$ we have

$$
\mu(x)=f^{\prime}(x)[\mu(f(x))]=\left(\operatorname{det} f^{\prime}(x)\right)^{-\frac{2}{n}} f^{\prime}(x)^{T} \mu(f(x)) f^{\prime}(x) .
$$

Iwaniec and Martin showed that for every uniformly quasiregular map $f$ there exists an $f$-invariant conformal structure [IM]; see also [Hi] for uniformly quasiregular semi-groups in two dimensions. We describe here all the possible invariant conformal structures for the chaotic Lattès type mappings and we will see that this is a very rigid situation.

Theorem 3. Let $f=f_{A}$ be one of the chaotic Lattès type mappings. Then a conformal structure $\mu$ is $f$-invariant if and only if the pull back of $\mu$ by the mapping $h$ is $M=h^{\prime}[\mu \circ h]$, a constant $\Gamma$ - and A-invariant structure. When the dimension $n \geq 3$, then $M$ has diagonal form:

$$
M=\operatorname{diag}\left(m_{1}, m_{2}, \ldots, m_{n}\right) \quad \text { with } \quad m_{i}>0 \quad \text { and } \quad m_{1} m_{2} \cdots m_{n}=1 .
$$

Remark. This result generalizes the following fact concerning the quasiconformal deformation of the Lattès rational mappings: when $A(z)=d z, z \in \mathbb{C}$, then any matrix $M \in S$ gives rise to an $f$-invariant conformal structure. In the case $A(z)=$ $\lambda z$ with $\lambda \in \mathbb{C} \backslash \mathbb{R}$ the $A$-invariance of $M$ forces it to be the identity. So we see that in the first case the Lattès rational map admits a (complex) 1-parameter family of quasiconformal deformations while in the second case the map does not admit any such deformation $[\mathrm{DH}]$.

Proof of Theorem 3. Let $f=f_{A}, A(x)=\lambda U x$, be a uniformly quasiregular chaotic Lattès type map and $\mu$ an $f$-invariant conformal structure. We pull back $\mu$ by $h$ and obtain $\nu(x)=h^{\prime}(x)[\mu(h(x))]$ for a.e. $x \in \mathbb{R}^{n}$. The new structure $\nu$ is $\Gamma_{2^{-}}$and $A$-invariant:

$$
A^{\prime}[\nu(A(x))]=A^{\prime}\left[h^{\prime}(A(x))[\mu(h \circ A(x))]\right]=h^{\prime}(x)\left[f^{\prime}(h(x))[\mu(f \circ h(x))]\right]
$$

and, using the $f$-invariance of $\mu$, we get, still for a.e. $x \in \mathbb{R}^{n}$,

$$
A^{\prime}[\nu(A(x))]=h^{\prime}(x)[\mu(h(x))]=\nu(x) .
$$


Since $\nu$ is a measurable function it is approximately continuous a.e. [Fe]. Let $x_{0}$ be a point where $\nu$ is approximately continuous: for every $\varepsilon>0$,

$$
\lim _{r \rightarrow 0} \frac{\left|\left\{x \in B\left(x_{0}, r\right) ; \operatorname{dist}\left(\nu\left(x_{0}\right), \nu(x)\right)<\varepsilon\right\}\right|}{\left|B\left(x_{0}, r\right)\right|}=1 .
$$

Let $\varepsilon>0$. For a given $\delta>0$ let $r>0$ so that

$$
\left|E_{0}\right|=\left|\left\{x \in B\left(x_{0}, r\right) ; \operatorname{dist}\left(\nu\left(x_{0}\right), \nu(x)\right)<\varepsilon\right\}\right| \geq(1-\delta)\left|B\left(x_{0}, r\right)\right| .
$$

We blow this up using the expanding map $A$. Denote

$$
x_{k}=A^{k}\left(x_{0}\right), \quad B_{k}=A^{k}\left(B\left(x_{0}, r\right)\right)=B\left(x_{k}, \lambda^{k} r\right) \quad \text { and } \quad E_{k}=A^{k}\left(E_{0}\right) .
$$

Clearly $\left|E_{k}\right| \geq(1-\delta)\left|B_{k}\right|$. Moreover, by the $A$-invariance of $\nu$,

$$
\operatorname{dist}\left(\nu\left(x_{k}\right), \nu\left(y_{k}\right)\right)=\operatorname{dist}\left(\left(A^{-k}\right)^{\prime}\left[\nu\left(x_{0}\right)\right],\left(A^{-k}\right)^{\prime}\left[\nu\left(y_{0}\right)\right]\right)=\operatorname{dist}\left(\nu\left(x_{0}\right), \nu\left(y_{0}\right)\right)<\varepsilon
$$

for every $y_{k}=A^{k}\left(y_{0}\right) \in E_{k}$. In other words,

$$
E_{k}=\left\{x \in B\left(x_{k}, \lambda^{k} r\right) ; \operatorname{dist}\left(\nu\left(x_{k}\right), \nu(x)\right)<\varepsilon\right\} .
$$

The group $S O(n)$ of orthogonal matrices is compact and thus a subsequence $U^{-k_{j}}$ converges to some $V \in S O(n)$. Let $M=V\left[\nu\left(x_{0}\right)\right]=\lim _{j \rightarrow \infty}\left(A^{-k_{j}}\right)^{\prime}\left[\nu\left(x_{0}\right)\right]$. Then $\operatorname{dist}(M, \nu(y)) \leq 2 \varepsilon$ for every $y \in E_{k}$, when $k=k_{j}$ is big enough.

Since $\nu$ is $\Gamma_{2}$-invariant we can project $E_{k}=E_{k}(\delta, \varepsilon)$ to the torus $\mathbb{T}$. In this way we get a set $\tilde{E}_{\delta, \varepsilon} \subset \mathbb{T}$ with $\left|\tilde{E}_{\delta, \varepsilon}\right| \geq(1-2 \delta)|\mathbb{T}|=1-2 \delta$, provided $k$ is big enough. The set $\tilde{E}_{\varepsilon}=\bigcup_{k \geq 3} \tilde{E}_{\frac{1}{k}, \varepsilon}$ has total mass $\left|\tilde{E}_{\varepsilon}\right|=1$ and, when $E_{\varepsilon}$ is the set above $\tilde{E}_{\varepsilon}$, $\operatorname{dist}(\nu(y), M) \leq 2 \varepsilon$ for every $y \in E_{\varepsilon}$. It suffices now to consider $\mathcal{E}=\bigcap_{k \geq 1} E_{\frac{1}{k}}$. This is a set of full measure such that $\operatorname{dist}(\nu(y), M)=0$ for every $y \in \mathcal{E}$. We conclude that $\nu$ is constant a.e.

Moreover, when the dimension $n \geq 3$, then an easy calculation, using the fact that $\nu$ has to be $\Sigma$-invariant, shows that $\nu$ is necessarily a diagonal matrix.

\section{UNIFORMLY QUASIREGULAR MAPS THAT BEHAVE LIKE POWER MAPPINGS}

Here we solve again Schröder's equation (3), this time using Zorich's map $h$. This can be done for affine mappings $A(z, t)=(\lambda z,|\lambda| t)$ respecting the reflections: $A \circ \Gamma_{1} \circ A^{-1} \subset \Gamma_{1}$; here $\lambda \in \mathbb{C},|\lambda|>1$ and we identified $x \in \mathbb{R}^{3}$ with $(z, t) \in \mathbb{C} \times \mathbb{R}$. For example, every multiplication $x \mapsto d x(d=2,3, \ldots)$ can be taken. In this way we obtain uniformly quasiregular mappings $f=f_{\lambda}$ of degree $|\lambda|^{2}$. In what follows we describe the properties of these mappings.

Proposition 5.1. The Julia set of $f=f_{\lambda}$ is $J(f)=\mathbb{S}^{2}$ and the complement of $J(f)$ consists of two components which are superattracting basins. The superattracting fixed points are 0 and $\infty$ and they are completely invariant.

Proof. Zorich's map $h$ sends the "ends" $-\infty, \infty$ of the cylinder $Z$ to $0, \infty$ respectively. By construction it is clear that $0, \infty$ are completely invariant and that $f$ is not locally homeomorphic at these points. The basins of attraction of these points are $h\left(\left\{x_{3}<0\right\}\right)=\mathbb{B}^{3}$ and $h\left(\left\{x_{3}>0\right\}\right)=\{|x|>1\}$ respectively. The remaining set $h\left(\left\{x_{3}=0\right\}\right)=\mathbb{S}^{2}$ is the Julia set of $f$, as in the proof of Lemma 3.2.

Proposition 5.2. The map $f$ can be conjugated by a quasiconformal map $\varphi$ which preserves $\mathbb{S}^{2}$ so that the restriction of $g=\varphi \circ f \circ \varphi^{-1}$ to $\mathbb{S}^{2}$ is the Lattès rational map induced by the multiplication $x \mapsto \lambda x$ and by the group $\Gamma_{1}$ restricted to $\left\{x_{3}=0\right\}$. 
In other words, the Lattès rational mappings in consideration can be extended to uniformly quasiregular mappings of $\overline{\mathbb{R}}^{3}$.

Proof. By construction $h:\left\{x_{3}=0\right\} \rightarrow \mathbb{S}^{2}$ is quasiregular. Let $\mu$ be the conformal structure obtained by pulling forward by $h: h^{\prime}[\mu \circ h]=I d$. The measurable Riemann mapping theorem assures the existence of a quasiconformal map $\varphi_{0}$ of $\mathbb{S}^{2}$ with $\varphi_{0}^{\prime}[I d]=\mu$. This map admits a quasiconformal extension $\varphi$ to $\mathbb{S}^{3}[\mathrm{TV}]$. Now, $\varphi \circ h$ restricted to $\left\{x_{3}=0\right\}$ is the (analytic) Weierstrass' $P$-function. Therefore, the restriction of $g=\varphi \circ f \circ \varphi^{-1}$ is the rational Lattès map associated to the multiplication $x \mapsto \lambda x$ and to the group $\Gamma_{1}$ restricted to $\left\{x_{3}=0\right\}$.

\section{REFERENCES}

[DH] A. Douady and J.H. Hubbard, A proof of Thurston's topological characterization of rational functions, Acta Math., 171 (1993), 263-297. MR 94j:58143

[Fe] H. Federer, Geometric measure theory, Springer-Verlag, Berlin, (1969). MR 41:1976

[Hi] A. Hinkkanen, Uniformly quasiregular semigroups in two dimensions, Ann. Acad. Sci. Fenn. Math. Vol. 21, (1996), 205-222. MR 96m:30029

[IM] T. Iwaniec and G. Martin, Quasiregular semigroups, Ann. Acad. Sci. Fenn. Math. Vol. 21, (1996), 241-254. MR 97i:30032

[MRV] O. Martio, S. Rickman and J. Väisälä, Topological and metric properties of quasiregular mappings, Ann. Acad. Sci. Fenn. Ser. A I, 488 (1971), 1-31. MR 45:8830

[MS] O. Martio and U. Srebro, Periodic quasimeromorphic mappings in $\mathbb{R}^{n}$, J. d'Analyse Math. 28 (1975), 20-40.

[Ri] S. Rickman, Quasiregular mappings, Springer-Verlag (1993). MR 95g:30026

[Sa] J. Sarvas, The Hausdorff dimension of the branch set of a quasiregular mapping, Ann. Acad. Sci. Fenn. Ser. A I Math. 1 (1975), 297-307. MR 53:805

[Tu] P. Tukia, On quasiconformal groups, J. d'Analyse Math., Vol. 46 (1986), 318-346. MR 87m:30043

[TV] P. Tukia and J.Väisälä, Quasiconformal extension from dimension $n$ to $n+1$., Ann. of Math. (2) 115 (1982), no.2, 331-348. MR 84i:30030

U.R.A. 751, UfR de Mathématiques Pures et Appliquées, Université de Lille I, 59655 Villeneuve D'AscQ, Cedex, France

E-mail address: mayer@gat.univ-lille1.fr 